\title{
Development and Evaluation of Bus Operation Control System Based on Cooperative Speed Guidance
}

\author{
Jing Teng and Weimin Jin \\ Key Laboratory of Road and Traffic Engineering, Ministry of Education, Tongji University, Shanghai 201804, China \\ Correspondence should be addressed to Weimin Jin; jwmemail01@126.com
}

Received 17 July 2014; Accepted 3 October 2014

Academic Editor: Yongjun Shen

Copyright (C) 2015 J. Teng and W. Jin. This is an open access article distributed under the Creative Commons Attribution License, which permits unrestricted use, distribution, and reproduction in any medium, provided the original work is properly cited.

\begin{abstract}
Buses often have strong bunching or large interval tendency when traveling further along the route. To restrain this further deterioration of operation service, this paper developed a bus operation control system to dynamically adjust bus speed, bus dwell time, and traffic signal timings along the running path. In addition, a simulation platform was developed to evaluate the proposed control system with the actual data collected from bus route number 210 in Shanghai. The simulation results show that the proposed control system can mitigate the amplification trend of the headway deviation along the route to produce headways within a given tolerance.
\end{abstract}

\section{Introduction}

The uncertainty of bus operation environments, such as the dynamic and stochastic traffic congestion and passenger demand, often causes the unreliability of bus operation service. On busy lines with short headways, buses usually arrive at stops irregularly, easily in bunches, which leads to the increase of passenger waiting time and the decrease of bus arrival punctuality rate. In China, bus transit system in the urban area is featured by the short frequency and no time-check points available on the bus route. There are several factors affecting the quality of bus operation service. With buses traveling further along the route, the effect of random factors will gradually be balanced while the effect of directional factors (e.g., weather condition, traffic congestion, and driver behavior) may become more and more significant, which eventually leads to bunching phenomenon. To alleviate the effect of directional factors, the recognition of a bus being late or early should be proactively predicted. In that way, the bus operators will have adequate time to restore the buses headway regularity. Besides, bus operators build slack into their schedules, which also leave room for the control. The development of advanced public transportation system (APTS), such as the automatic vehicle location (AVL) system and the automatic passenger count (APC) system, and the communication technologies makes the dynamic control of bus operation possible.

\section{Literature Review}

There are mainly two types of operation control strategies: the control strategy at stations and that on road sections. Holding control (holding buses at control stops) is a common effective method aiming at maintaining the planned bus schedule or headway [1-4], controlling the departure time to improve the stability of bus system. For non-frequently serviced bus route, target schedule control is taken, while for short frequently serviced bus route target headway control is used. Holding control strategies can be generally divided into two categories: one is the headway threshold-based control model in which buses are determined to hold at stops based on the deviation of their headway from the planned headway, and the other is holding times optimization model in which the holding time of each control stop is obtained through mathematical models aiming at minimizing the passengers waiting time $[2,5]$.

Single control point of holding control strategy is usually set in the high passenger demand stop or the middle section of the line [6]. Unfortunately, single-point control cannot succeed for long routes with frequent service and a strong bunching tendency [7]. Other point control includes the intersection signal control which is one of the effective ways to reduce bus headway fluctuation [8]. The speed control is continuous control method. Salek [9] used the Kalman filter forecasting models and proposed the idea of link speed control in different level. The fluctuation coefficient of the 
travel speed and smoothing coefficient of the driving were proposed $[10,11]$. Chen et al. proposed an adaptive cruise control system coordinated with the signal control strategy for BRT system [12].

Daganzo and Pilachowski [13] designed a control system with integration of cruise speed control and holding control under bus-bus cooperation condition based on dynamically predicting adjacent headway and proposed three combination control strategies to prevent buses from bunching. $\mathrm{Ma}$ et al. [14] proposed the economic-driving assistance system. The system can provide drivers with real-time bus speed and holding times, which makes the bus running state adapt to downstream intersection signal control and avoid bus stops at the intersections, thereby reducing fuel consumption and air pollution emissions.

The spatial variations of bus headway deviation can reflect the effect of directional factors. Based on analyzing the spatial variations of bus headway deviation, this paper proposed a proportional headway threshold. In addition, this paper proposed the bus operation control system based on cooperative speed guidance for the whole route. It relies on the cooperative speed control with other control strategies.

The remainder of this paper is organized as follows. First, the methodology (including the control principle, control threshold, control system architecture, and control algorithm) will be introduced. Then the performance of the proposed control will be evaluated by a case study using a simulation method. Finally, conclusion and future research will be addressed.

\section{Methodology}

3.1. Control Principle. The headway deviation from the departure terminal stop to the stop buses depart from (i.e., the difference between the departure headway of the stop and the scheduled headway) is defined as the path headway deviation. From the historical bus trajectories in the whole day (as shown in Figure 1), it is found that, with buses traveling further on the bus route, the variance of headway deviation tends to increase $[15,16]$. The headway deviation is so large (e.g., stop number 21008) that it is difficult to restore the downstream headway regularity with buses traveling further along the route. The single point control or small-scope control of the route seems not to restrain this strong bunching tendency successfully. From the perspective of the control target, it should reduce the headway deviation at the terminal stop (ensuring bus departure punctuality in the reverse direction) as well as some key midway stops (high passenger demand).

3.2. Proportional Control Threshold. The headway deviation at the terminal stop, some key midway stops, and some key intersection should fall within the tolerated scope. The red line marks the boundaries of bus deviation that would be slightly late or early from the scheduled headway deviation, as shown in Figure 1. The control threshold at each check node is given by

$$
\Delta h_{i}=t_{i} \frac{\Delta h_{d}}{t_{d}},
$$

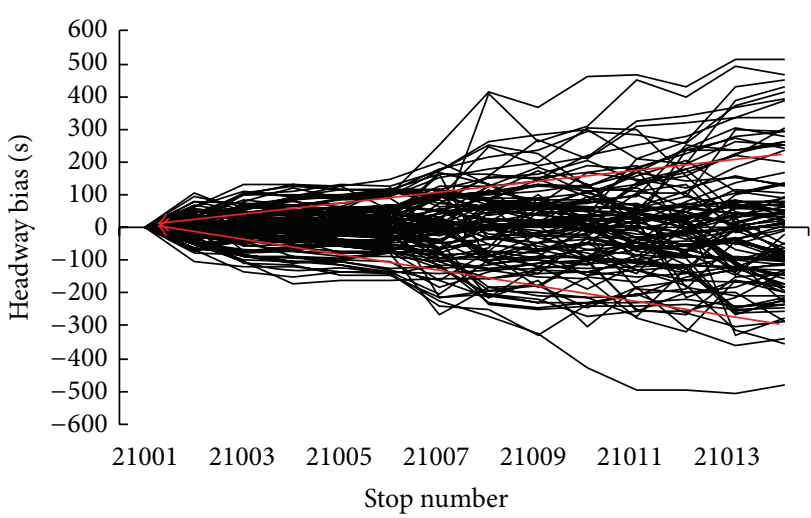

Figure 1: Headway deviation with buses traveling on the route (control unavailable).

where $\Delta h_{i}$ is the absolute value of control threshold at node $i$; $t_{i}$ is the path travel time at node $i ; \Delta h_{d}$ is the absolute value of allowed maximum headway deviation at the terminal stop; $t_{d}$ is the path travel time at the terminal stop.

3.3. Control System Architecture. Figure 2 illustrates the basic architecture of the proposed bus operation control system. This control system is regarded as the subsystem of bus operation dispatch system to maintain the established schedule. The signal control only is unreliable because drivers' behavior is unpredictable without speed adaption. On the contrary, speed adaption only also lacks stable effect due to the interaction from the other vehicles, which can be solved by signal control. Holding control cannot succeed for long routes with frequent service and a strong bunching tendency. Therefore, these three control strategies are simultaneously implemented to dynamically revise the fluctuation of driver behaviors in the control system. The system can dynamically adjust bus speed, bus dwell time, and traffic signal timings with buses traveling further along the route from the departure stop to destination stop. With the help of real-time communication between buses and operation center, the departure headway between adjacent buses can be collected and sent to the control center, when buses depart from the stop and the center can send the message to buses. Simultaneously, with the real-time communication between buses and the signal controllers, the buses can send request message to the signal controllers and the signal controllers can adjust signal timings.

3.4. Control Algorithm. As shown in Figure 3, the process of bus control system consists of four steps: (1) no action, (2) speed adjustment, (3) signal adjustment, and (4) delay notification. It takes no action if the detected headway deviation is less than the threshold at some key nodes (such as key intersection and key stop). If the detected headway deviation is beyond the threshold, the speed adjustment procedure is launched and the bus driver is notified. It is still possible that speed adjustment alone fails to bring the headway deviation back within the given tolerated scope. Then the signal timing adjustment procedure is triggered and 


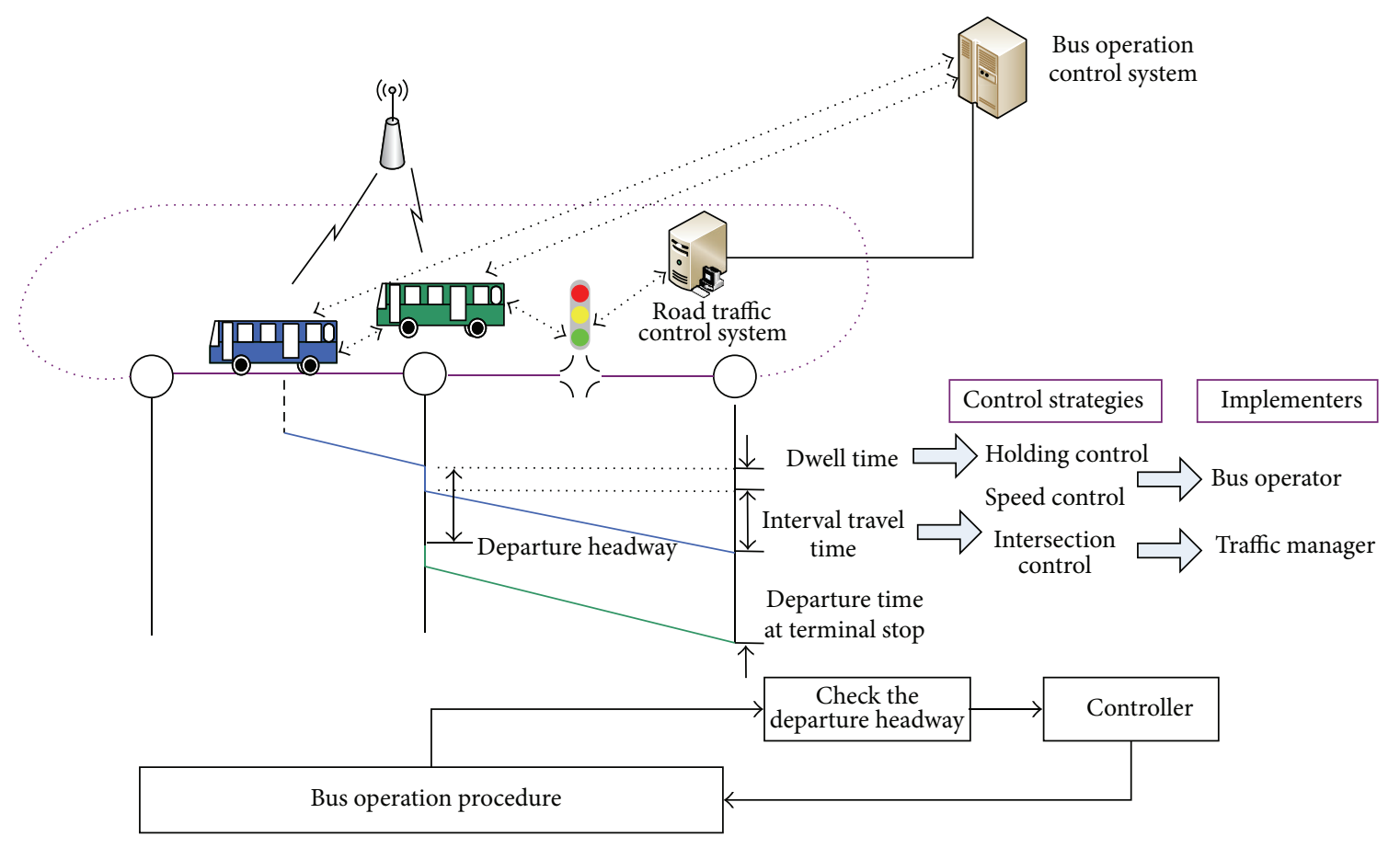

FIGURE 2: Control system architecture.

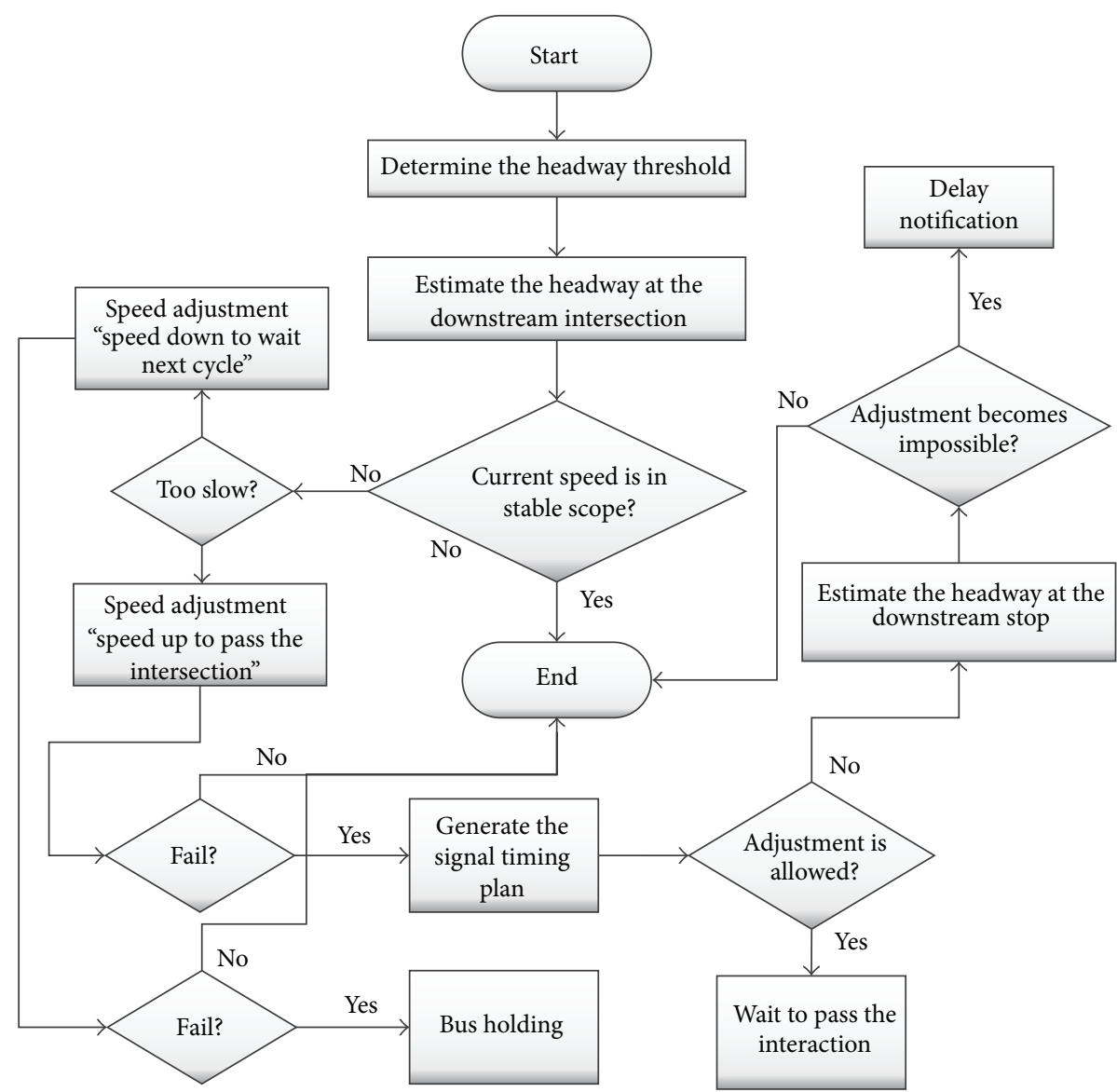

FIGURE 3: Flow chart of the control algorithm. 


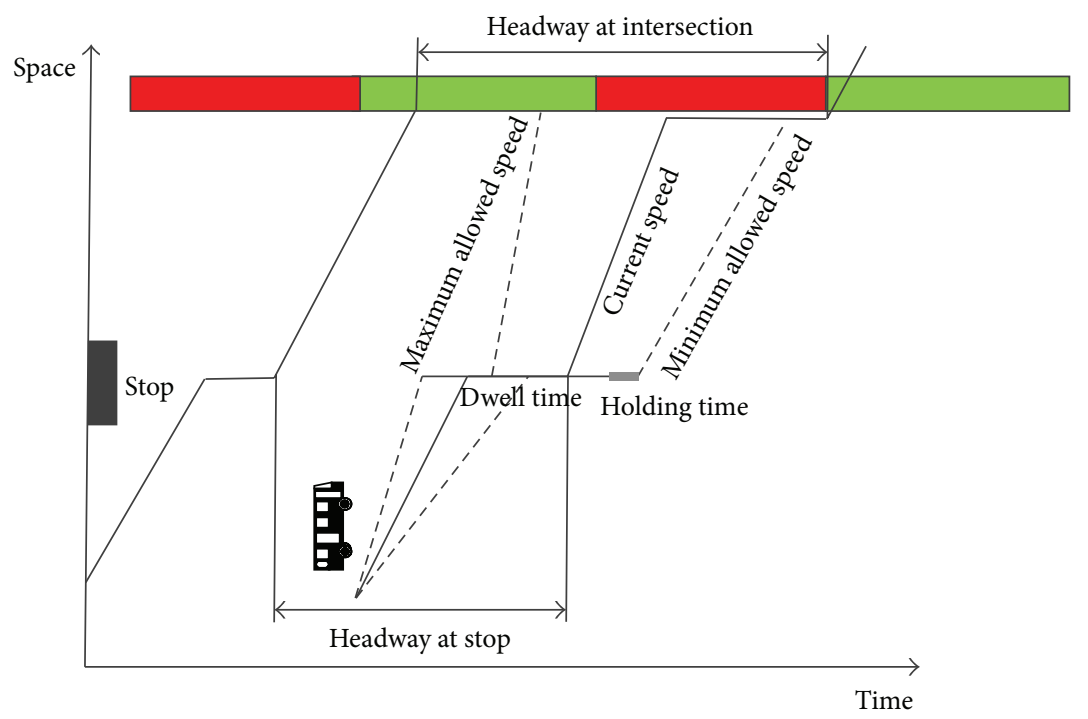

FIgURE 4: Speed adjustment procedure.

the bus' headway irregularity is reduced. If any appropriate adjustment becomes impossible, delay information would be notified to passengers waiting at the corresponding bus stops through electronic stop sign or mobile phone.

The speed adjustment procedure is the main component of control system, as shown in Figure 4. If the detected headway deviation at stop is less than the threshold, buses keep the current speed. Otherwise, buses will be instructed to change their current speeds. If the estimated headway deviation at intersection is less than the threshold, the signal timing plan will be maintained and the holding control will not be launched. Otherwise, the holding control and/or the signal control will be triggered. The speed adjustment is also appropriate to the control of early buses (Figure 4).

The speed is calculated in the two following scenarios.

(1) Speed Down to Wait Next Cycle. The time that the bus trip $i$ departs from the intersection $I$ is given by

$$
\operatorname{Dep}_{I}^{i}=\operatorname{Dep}_{S}^{i}+t_{j, j+1}^{i}+d_{S}^{i}+t_{H}=\operatorname{Dep}_{S}^{i}+\frac{L_{S, I}}{V^{\prime}}+d_{S}^{i}+t_{H} .
$$

The control threshold is determined by the following equation:

$$
\left(\operatorname{Dep}_{I}^{i}-\operatorname{Dep}_{I}^{i-1}\right)-\Delta P_{0}=\Delta h_{j}^{i}
$$

Substituting (2) into (3) leads to

$$
\left(\operatorname{Dep}_{S}^{i}+\frac{L_{S, I}}{V^{\prime}}+d_{S}^{i}+t_{H}-\operatorname{Dep}_{S}^{i-1}\right)-\Delta P_{0}=\Delta h_{S}^{i}
$$

The departure time at intersection falls within the green phase of the current cycle, $t_{\mathrm{sg}} \leq \operatorname{Dep}_{S}^{i}+\left(L_{S, I} / V^{\prime}\right)+d_{S}^{i}+t_{H} \leq t_{\mathrm{eg}}$, $V^{\prime}=L_{S, I} /\left(\Delta h_{S}^{i}-\operatorname{Dep}_{S}^{i}-d_{S}^{i}-t_{H}+\operatorname{Dep}_{S}^{i-1}+\Delta P_{0}\right)$.
The adjusted speed of the link is given by

$$
V_{j, j+1}=\left\{\begin{array}{l}
V_{j, j+1}^{\min }, \quad V^{\prime}<V_{j, j+1}^{\min } \\
\frac{L_{S, I}}{\left(\Delta h_{S}^{i}-\operatorname{Dep}_{S}^{i}-d_{S}^{i}-t_{H}+\operatorname{Dep}_{S}^{i-1}+\Delta P_{0}\right)}, \\
\min \left\{V_{S, I}^{\min }, \frac{L_{S, I}}{t_{\mathrm{eg}}-\operatorname{Dep}_{S}^{i}-d_{S}^{i}-t_{H}}\right\} \\
\leq V^{\prime} \leq \max \left\{V_{S, I}^{\max }, \frac{L_{S, I}}{t_{\mathrm{sg}}-\operatorname{Dep}_{S}^{i}-d_{S}^{i}-t_{H}}\right\} .
\end{array}\right.
$$

(2) Speed Up to Pass the Intersection. The time that the bus trip $i$ departs from the intersection $I$ is given by

$$
\operatorname{Dep}_{I}^{i}=\operatorname{Dep}_{S}^{i}+t_{j, j+1}^{i}+d_{S}^{i}=\operatorname{Dep}_{S}^{i}+\frac{L_{S, I}}{V^{\prime}}+d_{S}^{i} .
$$

The control threshold is determined by the following equation:

$$
\left(\operatorname{Dep}_{I}^{i}-\operatorname{Dep}_{I}^{i-1}\right)-\Delta P_{0}=\Delta h_{j}^{i} .
$$

Substituting (6) into (7) leads to

$$
\left(\operatorname{Dep}_{S}^{i}+\frac{L_{S, I}}{V^{\prime}}+d_{S}^{i}-\operatorname{Dep}_{S}^{i-1}\right)-\Delta P_{0}=\Delta h_{S}^{i}
$$

The departure time at intersection falls within the green phase of the current cycle, $t_{\mathrm{sg}} \leq \operatorname{Dep}_{S}^{i}+\left(L_{S, I} / V^{\prime}\right)+d_{S}^{i} \leq t_{\mathrm{eg}}, V^{\prime}=$ $L_{S, I} /\left(\Delta h_{S}^{i}-\operatorname{Dep}_{S}^{i}-d_{S}^{i}+\operatorname{Dep}_{S}^{i-1}+\Delta P_{0}\right)$. 


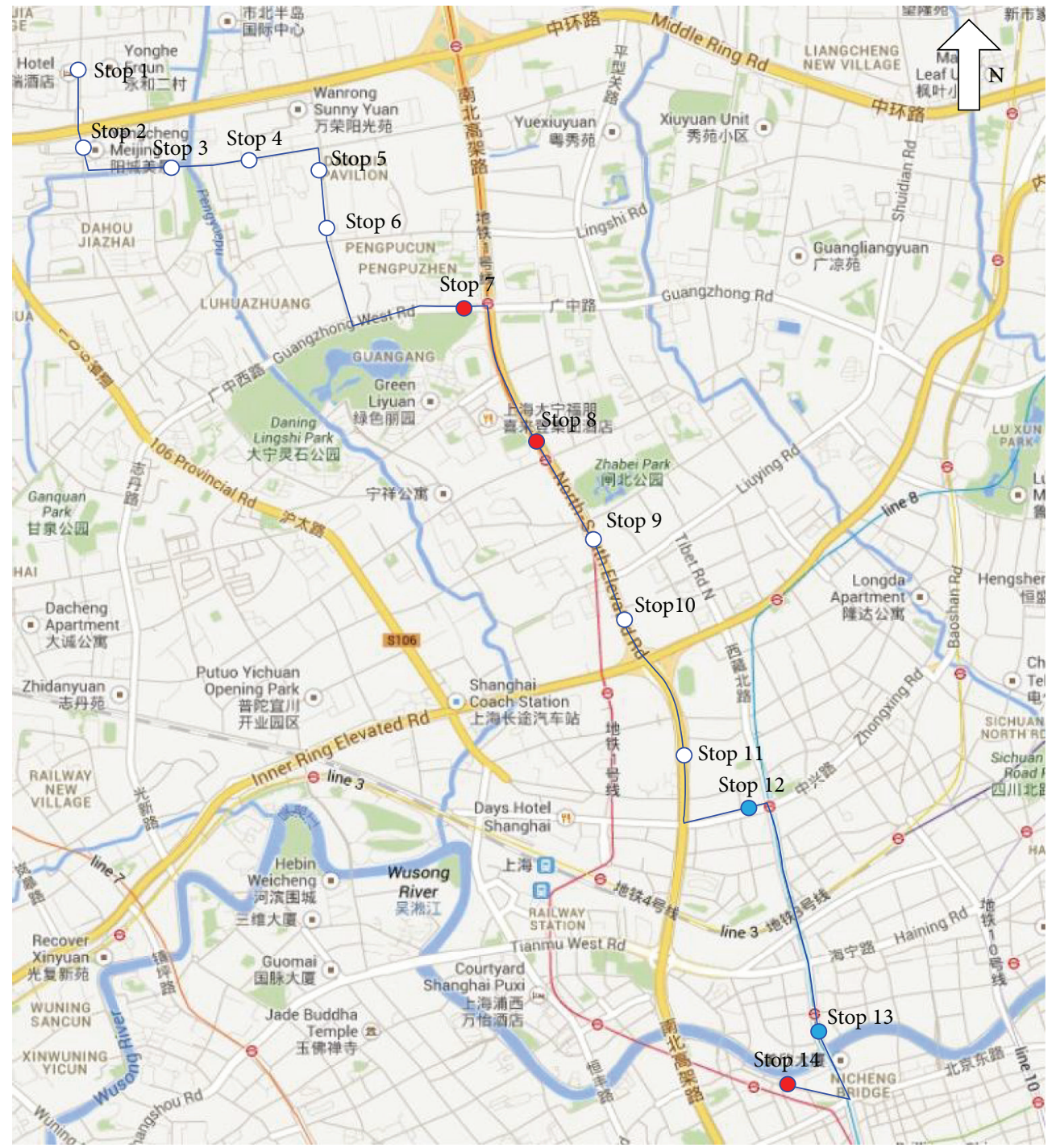

Stop name
(1) Yonghe Village
(2) Gaopin/Wenshui
(3) Yonghe/Yuanping
(4) Yonghe/Wanrong District
(5) Wanrong/Yonghe
(6) Wanrong/Lingshi
(7) Guangzhong West/Gonghexinlu
(8) Gonghexinlu/Yanchang
(9) Zhabei Park
(10) Gonghexinlu/Zhongshan North
(11) Gonghexinlu/Zhonghuaxinlu
(12) Zhongxing/Xizang North

(13) Xizang North/Qufu

(14) Xinzha/Wenzhou

Regular stop

Transfer stop with line 1

Transfer stop with line 8

FIGURE 5: Bus route number 210 located in Shanghai.

The adjusted speed of the link is given by

$$
V_{j, j+1}=\left\{\begin{array}{l}
V_{j, j+1}^{\max }, \quad V^{\prime}>V_{j, j+1}^{\max } \\
\frac{L_{S, I}}{\left(\Delta h_{S}^{i}-\operatorname{Dep}_{S}^{i}-d_{S}^{i}+\operatorname{Dep}_{S}^{i-1}+\Delta P_{0}\right)}, \\
\min \left\{V_{S, I}^{\min }, \frac{L_{S, I}}{t_{\mathrm{eg}}-\operatorname{Dep}_{S}^{i}-d_{S}^{i}}\right\} \\
\leq V^{\prime} \leq \max \left\{V_{S, I}^{\max }, \frac{L_{S, I}}{t_{\mathrm{sg}}-\operatorname{Dep}_{S}^{i}-d_{S}^{i}}\right\},
\end{array}\right.
$$

where $V_{S, I}$ is the recommended speed of the link between stop $S$ and intersection $I ; V_{S, I}^{\max }$ is the maximum allowed speed of the link between stop $S$ and intersection $I ; V_{S, I}^{\min }$ is the minimum allowed speed of the link between stop $S$ and intersection $I ; \Delta P_{0}$ is the headway of the departure terminal stop; $\Delta h_{I}^{i}$ is the value of control threshold at intersection $I ; t_{H}$ is the holding time at the stop, $t_{H} \leq t_{H}^{\max } ; t_{\mathrm{sg}}$ is the start time of green phase; $t_{\mathrm{eg}}$ is the end time of green phase; $L_{S, I}$ is the distance between stop $S$ and intersection $I ; t_{j, j+1}^{i}(j \geq 2, i \geq 2)$ is the link travel time; $d_{S}^{i}$ is the dwell time at stop $S$; $\operatorname{Dep}_{S}^{i}$ is the time that bus trip $i$ departs from stop $S$; $\operatorname{Dep}_{I}^{i}$ is the time that bus trip $i$ departs from intersection $I$. 


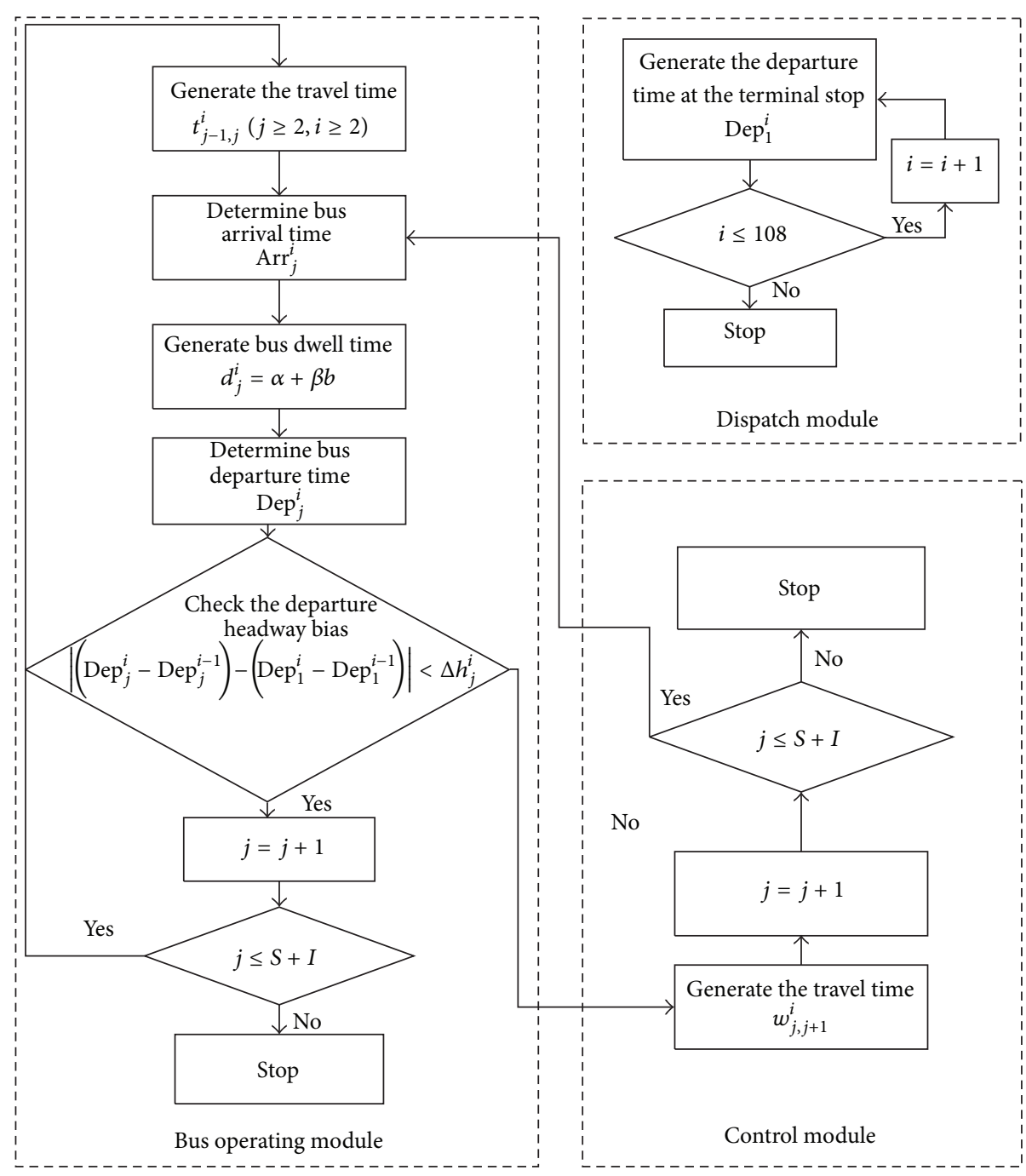

Figure 6: Flowchart of transit simulation procedure.

\section{Case Study}

4.1. Experimental Data. The experimental data of bus operating was obtained from the AVL data of bus route number 210 located in Shanghai, AVL records from April 15, 2013, to September 15, 2013, (5:00 am-10:00 pm). As shown in Figure 5 , the southbound corridor has 14 stops and is 9.75 kilometers long, from Yonghe Village stop to Xinzha/Wenzhou stop. The route is frequently serviced with the scheduled headway of 35 minutes during peak periods and 7-10 minutes during offpeak periods. Passenger data was obtained from field surveys.

4.2. Simulation Process. The simulation updates the locations of all buses in sequence at regular simulation time steps. The simulation model includes three subcomponents: the dispatch module, the bus operating module, and the control module. Figure 6 presents the simulation process of the buses. The simulation procedure describes the bus operational status: departure from the start stop, travelling on the road, arrival at the stop, and departure from the stop, serving passengers at the stop and adjusting speed on the road.

The bus operating module simulates the operation process of each bus as well as its operating environment. Bus travel time is usually simulated using normal and lognormal distribution [5]. Through statistical analysis of historical data, it is found that bus travel time follows normal distribution. Based on the generated travel time, the bus arrival time at immediate-downstream stop can be determined. Besides, through statistical analysis of the field data, passengers arrive at the bus station randomly, following the Poisson distribution. For the frequently serviced bus lines (i.e., the scheduled headway of less than 10 minutes), this assumption is reasonable. The dwell time is assumed to be a linear function of boarding count, $b$, the equation is given by $d_{j}^{i}=\alpha+\beta b$, and the parameters $\alpha, \beta$ can be specified from the experimental data. After determining the dwell time, the time that bus departs from the stop, $\operatorname{Dep}_{j}^{i}$, is derived. The signal timing plans of each intersection are generated one cycle by one cycle 


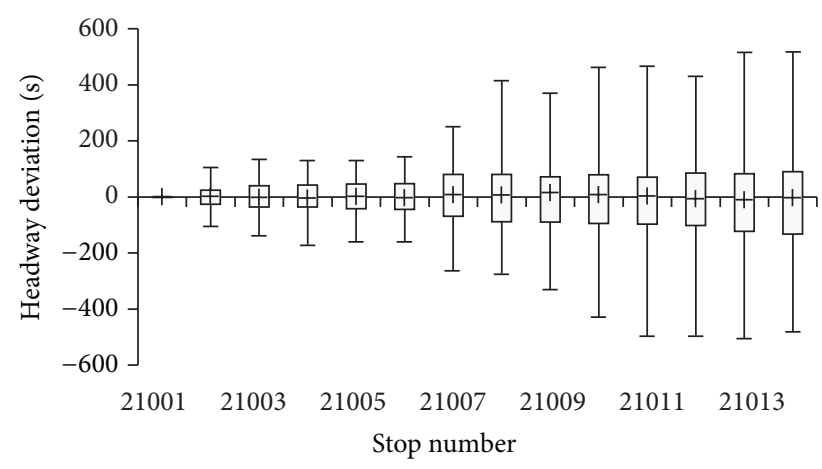

(a) Control unavailable

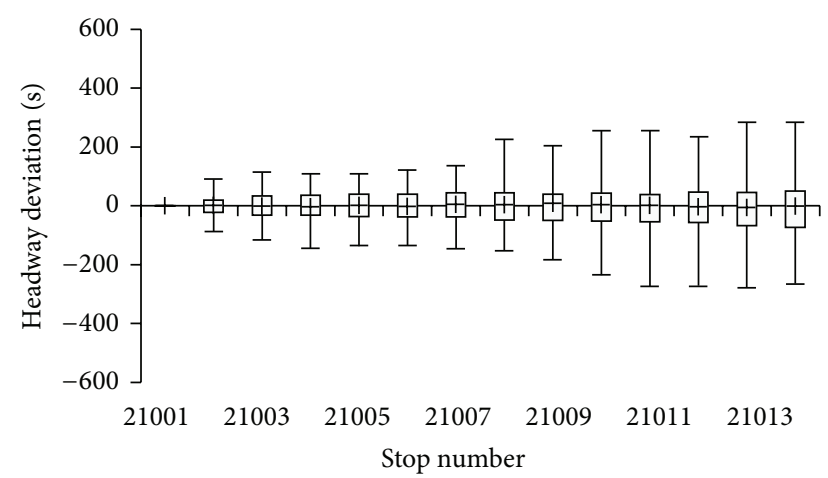

(b) After control

Figure 7: Distribution of headway deviation with buses traveling on the route.

when the simulation starts. The starting time and ending time of green phase will be updated in simulation step.

The control module simulates the adjustment bus speed process. Before generating the next link travel time (speed), it will check whether headway deviation between adjacent buses is within the given range. If not, it will change the bus speed and regenerate the next link travel time (speed).

When a complete simulation process of the bus trip is finished, the dispatch module will generate the departure time of next trip at the start stop according to the scheduled timetable.

4.3. Measures of Effectiveness. Three performance measures are used to compare different control strategies: the headway variance, the passenger waiting time, and the travel time. The travel time can reflect the efficiency of the system, while the headway variance, calculated for all trips and stops, can reflect the system stability.

4.3.1. Headway Variance. As shown in Figure 7, when bus control is not available, bus headway deviation at the beginning of several stops varies little. However, with buses traveling further on the bus route, the variance of headway deviation tends to increase. When the proposed control system is implemented, the upper and the lower boundaries of the headway deviation are decreased in some extent. The possible reasons for this may be that, at the first several stops, the proposed control system promptly corrects the irregularity of bus

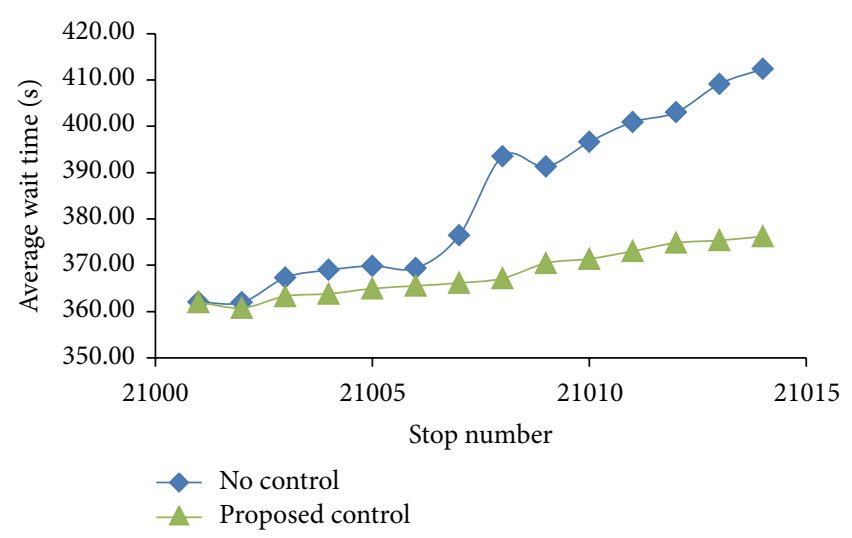

FIGURE 8: Impact of bus control on average wait time.

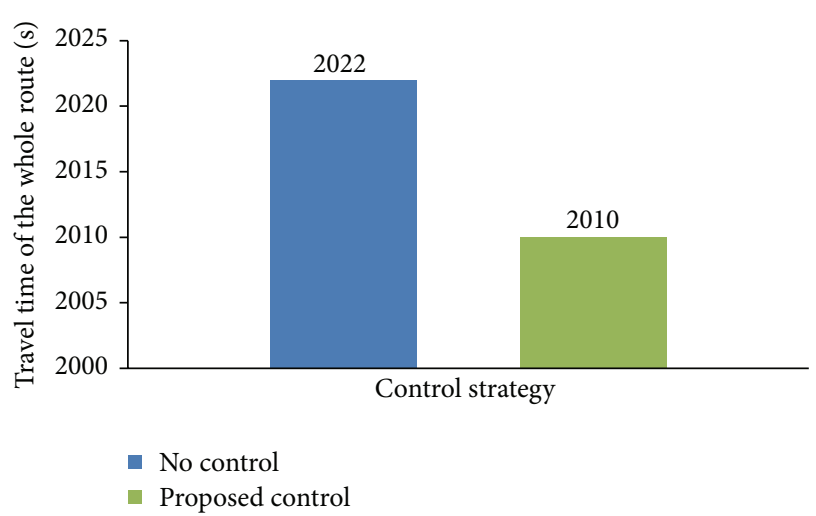

FIGURE 9: Comparison of travel time in different control strategies.

headway, reducing the bus headway deviation amplification trend.

4.3.2. Passenger Waiting Time. As shown in Figure 8, the control system can decrease the average wait time considerably. With buses traveling further on the route, the average wait time varies little.

4.3.3. Bus Travel Time. As shown in Figure 9, the bus travel time decreases slightly when the control system is implemented. The system improves the regularity of the headway at the cost of travel time of some trips. The faster trips and the slower trips are balanced after control to a certain extent.

\section{Conclusion and Future Work}

This paper proposed a proportional headway threshold model, which is a metric of the dynamic speed guidance. The bus operation control system integrates cooperative control strategies to reduce the accumulation of the headway deviation and through the experiment case, it could be found that the control system can improve the stability and the efficiency of the bus fleet and save the travel cost of the passengers.

It is noted that the purpose of the current research is to improve the bus headway regularity of the route and the signal timing adjustment procedure does not consider the potential delays of other vehicles traveling opposite to bus at 
intersection. We will refine our control strategy if such delays are serious.

\section{Conflict of Interests}

The authors declare that there is no conflict of interests regarding the publication of this paper.

\section{Acknowledgments}

This work was supported by the National Natural Science Foundation of China (Grant no. 61174185).

\section{References}

[1] J. G. Strathman, T. J. Kimpel, K. J. Dueker et al., "Bus transit operations control: review and an experiment involving TriMet's automated bus dispatching system," Journal of Public Transportation, vol. 4, 2001.

[2] E. E. Osuna and G. F. Newell, "Control strategies for an idealized public transportation system," Transportation Science, vol. 6, no. 1, pp. 52-72, 1972.

[3] J. Argote, Y. Xuan, and V. Gayah, "Comparative analysis of various bus control strategies: a case study of the UC Berkeley Bear Transit System," in Proceedings of the 12th Conference on Advanced Systems for Public Transport, Santiago, Chile, July 2012.

[4] Y. Ji and H. M. Zhang, "Dynamic holding strategy to prevent buses from bunching," Transportation Research Record: Journal of the Transportation Research Board, vol. 3, no. 2352, pp. 94103, 2013.

[5] D. Koffman, "A simulation study of alternative real-time bus headway control strategies," Transportation Research Record, no. 663, pp. 41-46, 1978.

[6] L. Fu and X. Yang, "Design and implementation of bus holding control strategies under real-time information," Transportation Research Record: Journal of the Transportation Research Board, no. 1791, pp. 6-12, 2002.

[7] C. F. Daganzo, "A headway-based approach to eliminate bus bunching: Systematic analysis and comparisons," Transportation Research B: Methodological, vol. 43, no. 10, pp. 913-921, 2009.

[8] A. Adamski and A. Turnau, "Simulation support tool for realtime dispatching control in public transport," Transportation Research Part A, vol. 32, no. 2, pp. 73-87, 1998.

[9] S. Salek, "A method for predicting the mean and variance of transit segment and route travel times," in Transportation Research Record: Journal of the Transportation Research Board, No. 2256, pp. 30-37, Transportation Research Board of the National Academies, Washington, DC, USA, 2011.

[10] Y. Ding and S. Chien, "Improving transit service quality and headway regularity with real-time control," Transportation Research Record, no. 1760, pp. 161-170, 2001.

[11] Z. R. Peng, E. D. Lynde, and W. Y. Chen, Improving Service Restoration Using Automatic Vehicle Location, Midwest Regional University Transportation Center, College of Engineering, Department of Civil and Environmental Engineering, University of Wisconsin, Madison, Wis, USA, 2008.

[12] Q. Chen, E. Adida, and J. Lin, "An investigation in real-time bus holding policy," in Proceedings of the 15th Hong Kong Society of Transportation Study International Conference (HKSTS '10),
Hong Kong Society for Transportation Studies, Hong Kong, China, December 2010.

[13] C. F. Daganzo and J. Pilachowski, "Reducing bunching with busto-bus cooperation," Transportation Research B: Methodological, vol. 45, no. 1, pp. 267-277, 2011.

[14] W. Ma, H. Xie, and B. Han, "Development and evaluation of an economic-driving assistance program for transit vehicles," Energies, vol. 5, no. 2, pp. 371-385, 2012.

[15] G. Chen, J. Teng, S. Zhang, and X. Yang, "Tendency-based approach for link travel time estimation," Journal of Transportation Engineering, vol. 139, no. 4, pp. 350-357, 2013.

[16] G. Chen, X. Zhou, D. Zhang, and X. Yang, "Proportion-based and tendency-based bus trajectory prediction models," Journal of Transportation Engineering, vol. 139, no. 9, pp. 896-902, 2013. 


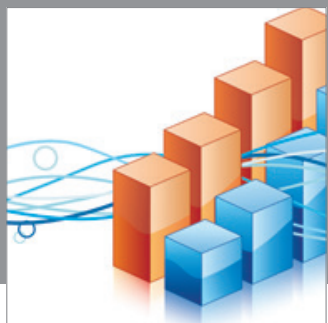

Advances in

Operations Research

mansans

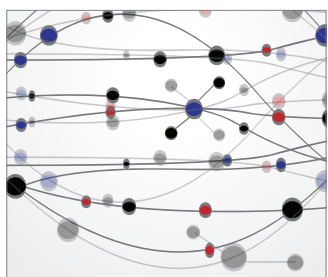

The Scientific World Journal
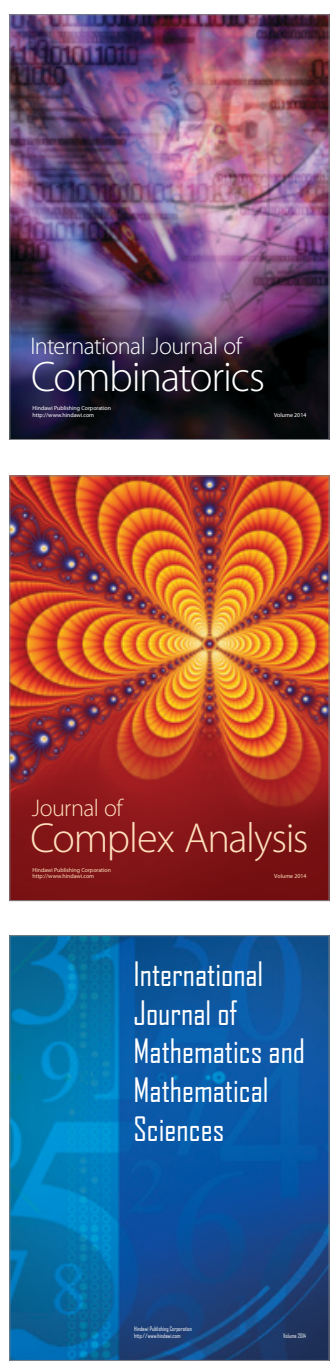
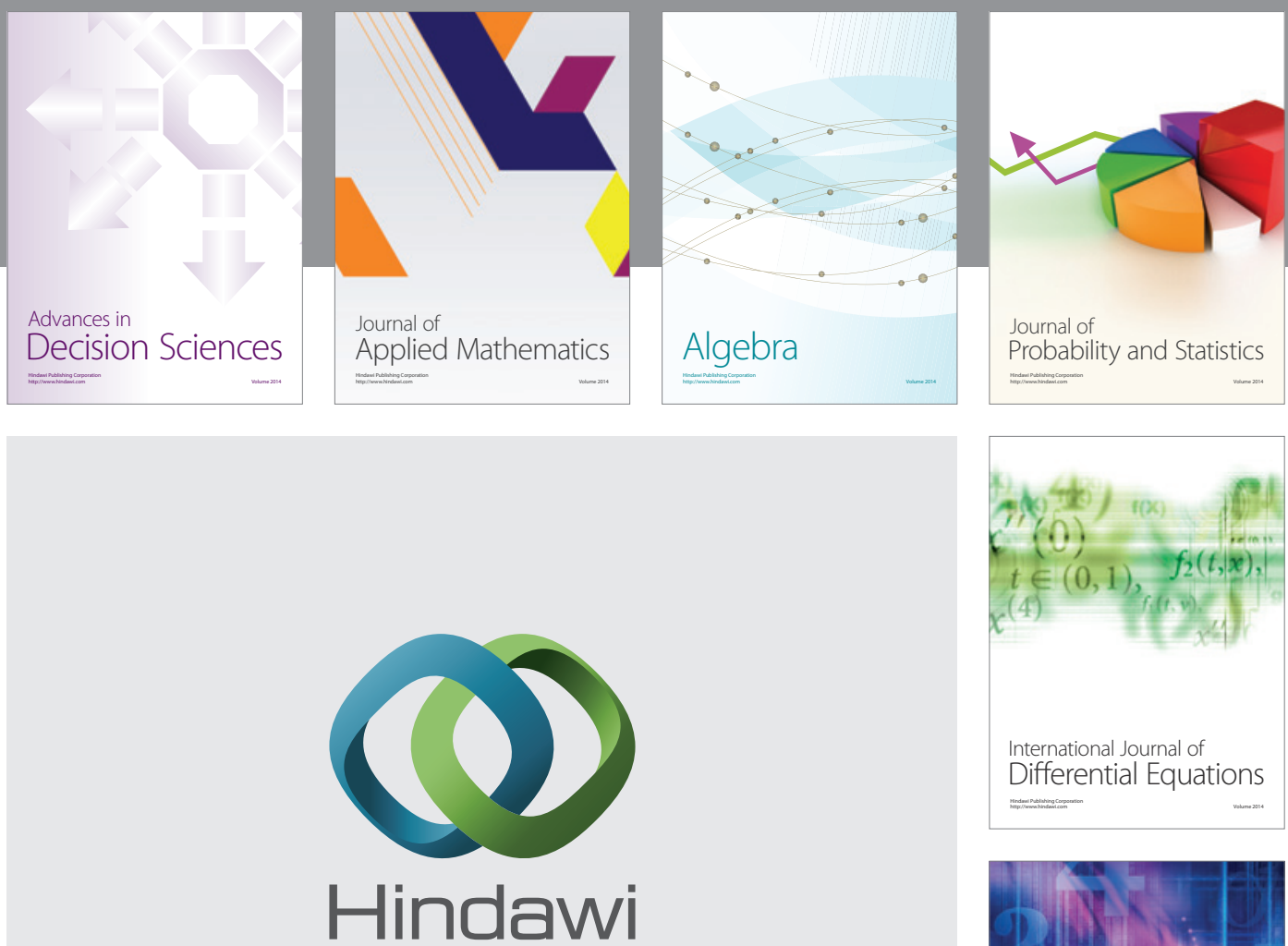

Submit your manuscripts at http://www.hindawi.com
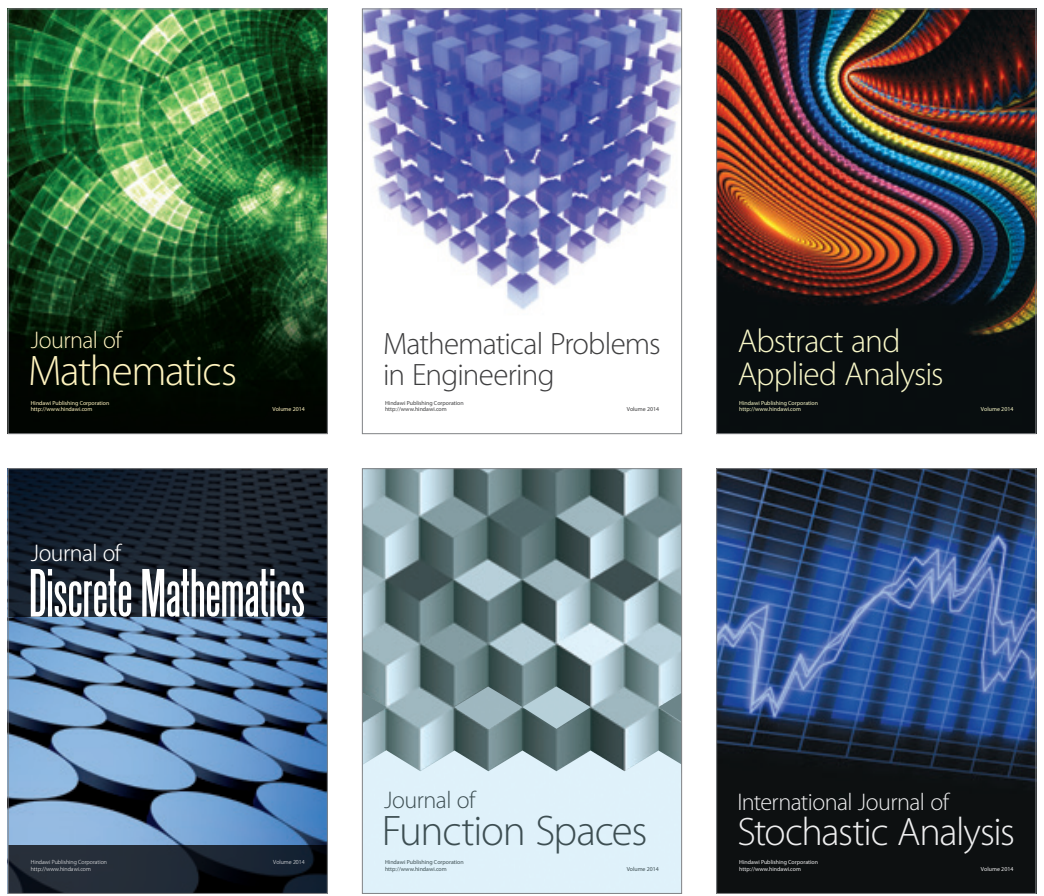

Journal of

Function Spaces

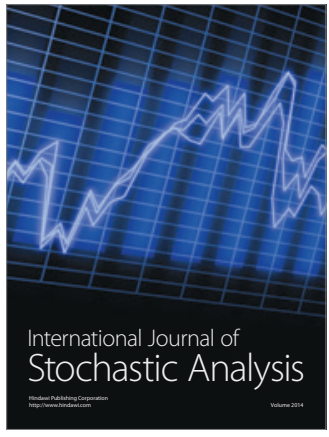

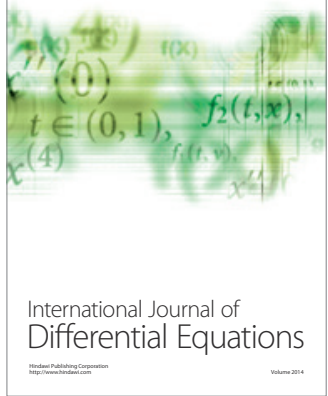
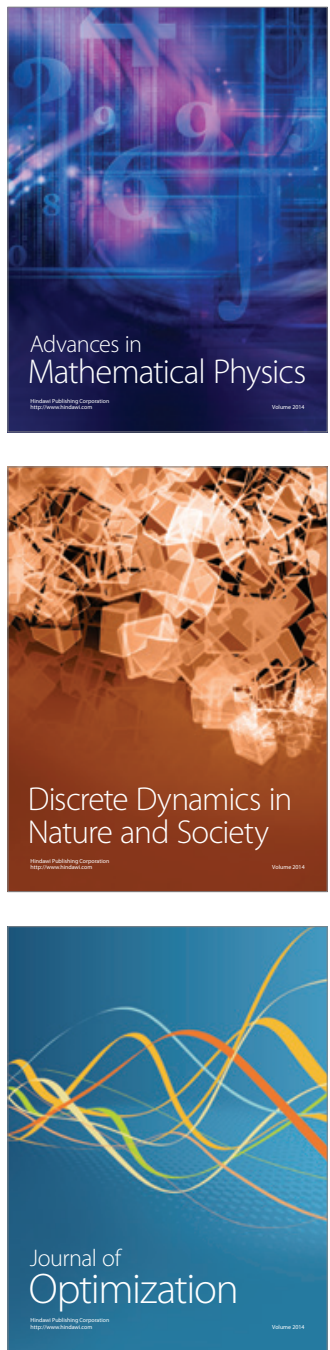